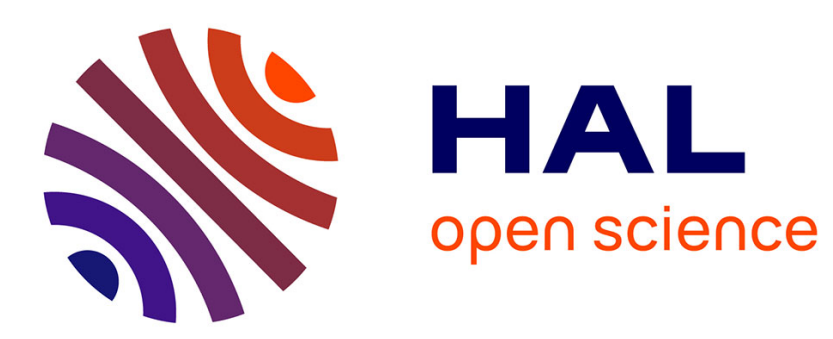

\title{
Hydrodynamic modelling of complex fixed bed geometries in simulated moving bed adsorption processes
}

Leonel Fangueiro Gomes, Frédéric Augier, Damien Leinekugel-Le-Cocq, Ivana Vinkovic, Serge Simoëns

\section{To cite this version:}

Leonel Fangueiro Gomes, Frédéric Augier, Damien Leinekugel-Le-Cocq, Ivana Vinkovic, Serge Simoëns. Hydrodynamic modelling of complex fixed bed geometries in simulated moving bed adsorption processes. Chemical Engineering Science, 2015, 132, pp.46-58. 10.1016/j.ces.2015.04.019 . hal-01174858

\section{HAL Id: hal-01174858 \\ https://hal.science/hal-01174858}

Submitted on 10 Jul 2015

HAL is a multi-disciplinary open access archive for the deposit and dissemination of scientific research documents, whether they are published or not. The documents may come from teaching and research institutions in France or abroad, or from public or private research centers.
L'archive ouverte pluridisciplinaire HAL, est destinée au dépôt et à la diffusion de documents scientifiques de niveau recherche, publiés ou non, émanant des établissements d'enseignement et de recherche français ou étrangers, des laboratoires publics ou privés. 


\title{
Hydrodynamic modelling of complex fixed bed geometries in Simulated Moving Bed adsorption processes
}

\author{
L. Fangueiro Gomes ${ }^{\mathrm{a}, \mathrm{b}}$, F. Augier ${ }^{\mathrm{a},{ }^{*}}$, D. Leinekugel-le-Cocq ${ }^{\mathrm{a}}$, I. Vinkovic ${ }^{\mathrm{b}}$, S. Simoëns ${ }^{\mathrm{b}}$ \\ * Corresponding author. Tel.: +33 4780221 42; fax: +33 478022008 . \\ E-mail address: frederic.augier@ifpen.fr (F. Augier). \\ IFP Energies nouvelles, Rond-point de l'échangeur de Solaize, BP3, 69360 Solaize, France \\ LMFA, UMR CNRS 5509, Ecole Centrale de Lyon, Université de Lyon I, INSA Lyon, 69131, Ecully \\ Cedex, France
}

\begin{abstract}
Hydrodynamics inside industrial Simulated Moving Bed (SMB) adsorption columns can be complex due to the presence of internal distribution devices. They have to be taken into account in SMB numerical models to scale-up processes. In the present work, CFD is used as an intermediate step to develop a 1D model simple enough to be used for cyclic SMB simulations while being able to represent realistic hydrodynamics. First, a mock-up representative of an industrial SMB is used to perform Residence Time Distribution (RTD) experiments and to provide validation data. Experiments are well predicted by a CFD model including porous media and turbulent zones, allowing to consider CFD simulations as references to fit simpler models. The moments of internal age distribution are characterized following the calculation method developed by Liu and Tilton (2010), which allows to estimate the degree of mixing (Liu, 2012) inside adsorption beds. A major result is that RTD and degree of mixing inside adsorption beds are well described by a 1D multi-exit model, unlike classical dispersed plug flow models (Ruthven, 1989) that were generally used to simulate SMB processes. Additionally, a numerical method was developed which is able to reproduce the RTD with steady state simulations.
\end{abstract}

\section{Keywords:}

Simulated Moving Bed, Adsorption, CFD, RTD, Stationary RTD, Degree of Mixing

\section{INTRODUCTION}

\subsection{SMB processes}

Simulated Moving Bed (SMB) is a cyclic adsorption process used to separate molecules that are hardly separable by distillation, solvent extraction or other classical operation units (Ruthven and Ching, 1989). Several industrial applications are known. A classical one is the separation of xylene isomers (Minceva and Rodrigues, 2007; Bergeot et al., 2010; Laroche et al., 2011) operated in adsorption columns up to 10 meters of diameter. SMB comprises a set of superposed fixed bed adsorbers which present several hydrodynamic heterogeneities, as pipes and beams placed in the porous media, the packing itself and non-ideal injection and collecting devices, as shown in Fig. 1. Such heterogeneities generate significant deviations from the desired plug flow. As far as transport limitations inside (diffusion) or outside (external mass transfer) adsorbent particles generate major dispersion in the process, the impact of hydrodynamic discrepancies is not significant. However, when low dispersive adsorption media is used, hydrodynamics can become the most dispersive phenomenon of the process. In this context, it is crucial to study correctly hydrodynamics inside adsorption beds and its effect on adsorption performances. 


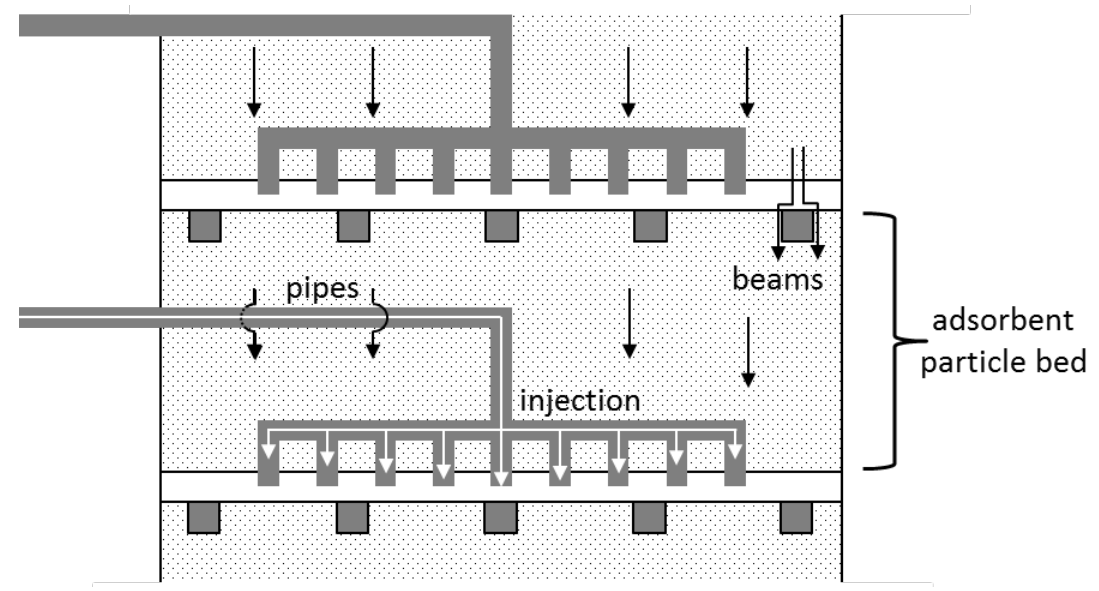

Fig. 1 - Schematic view of an example of one SMB geometry.

\subsection{Residence Time Distribution and CFD}

In order to model industrial processes, a classic approach for chemical engineers consists in studying the RTD (Danckwerts, 1953) and estimating the global axial dispersion resulting from internal flow patterns (Van Baten et al., 2001; Moustiri et al., 2001). Despite being a very powerful tool for hydrodynamics characterization, the RTD only gives overall information and does not take into account the quality of the mixing or dispersive phenomena in the flow. The lack of information on internal hydrodynamics can lead to inappropriate modelling and important errors on the calculation of performances. For this reason, Danckwerts (1958) and Zwietering (1959) introduced the concept of Degree of Mixing $(J)$. Zwietering showed for example that reactors with the same RTD but different $J$ can lead to different performances in case of chemical reactions with non-linear kinetics (for instance, of order $n \neq 1$ ). Other studies have been recently done to fill in the gaps of classical RTD methods, as Smart RTD (Simcik et al. 2012) and the reactive-mixing index analysis (Yablonsky et al., 2009). Yet, Claudel et al. (2002) and Hocine et al. (2008) developed simple hydrodynamic models through the RTD by making some assumptions about the internal information of the systems in study.

However, in many applications, including adsorption processes, RTD has been extensively used to estimate overall axial dispersion coefficients. Dispersion coefficients are then used in dispersed plugflow (DPF) models to take into account the effect of hydrodynamics on the separation (Ruthven, 1989). When low dispersion coefficients are involved, DPF models are very close to a cascade of $N$ Continuous Stirred-tank Reactors (NCSTR). In the following, DPF and NCSTR models are considered as equivalent $(P e=2 N$ for $P e>50$, Schweich, 2001). Augier et al. (2008) estimated the impact of obstacles within porous media on the dispersion of concentration fronts by measuring the resulting variance, allowing to further adjust equivalent DPF models. Notwithstanding, asymmetric RTD curves were already found, pointing out the relative unsuitability of DPF models, which may lead to strong discrepancies in the scenario discussed above. Kwapinski et al. (2010) had already shown the inadequacy of the plug flow model for the representation of the impact of adsorber packed bed hydrodynamics and thermal effects on the breakthrough curves.

Otherwise, CFD has well been known for more than 20 years as a pragmatic and reliable tool to describe local hydrodynamics in various reactors and separation processes. It can be used as a "stand-alone" numerical tool to solve simultaneously hydrodynamics, transfer and kinetics at any point of a geometry. This direct approach can be very time consuming. Zheng et al. (2010) performed CFD simulations to study a Pressure Swing Adsorption cyclic process successfully. In the present SMB processes, it is still unrealistic to consider it. A more rational method consists in studying hydrodynamics with CFD and deducing a simpler hydrodynamic model that can be easily coupled with other physics. This last method is the one used in the present work. CFD can be used for example to 
simulate RTD. This kind of calculation is important but fastidious because long physical times have to be solved to simulate the transport of a passive tracer inside the process, and lastly does not give quantitative information on internal mixing phenomenon as previously pointed out. Recently, Liu (2012) proposed to use CFD to calculate various internal information inside reactors. Based on the transport of the first and second moments of the internal age distribution, Liu developed a method to calculate the Degree of Mixing $J$ introduced five decades ago but used, up to now, as a theoretical concept. Furthermore, this method is based on steady-state, i.e. inexpensive CFD simulations.

The method is sufficient to estimate the variance ( $2^{\text {nd }}$ moment) of a RTD, and then the equivalent number of CSTR $N$. Nevertheless it is hardly usable to reconstruct the RTD curve. Indeed, the reconstruction of a distribution from its moments is an impossible task since even the knowledge of all the moments up to infinity can lead to different functions (John et al. 2007).

\subsection{Objectives of the study}

Based on the recent advances of flow characterisation by CFD, it is proposed to characterize in detail hydrodynamics of a SMB process, and then to deduce a simple but robust 1D model that can be used in cyclic simulations. For this, a new method is proposed that can, under some assumptions, calculate RTD only by transporting the first and second moments of the internal age inside the studied configuration.

The study is divided in four steps:

- RTD experiments in a laboratory setup representative of a SMB fixed bed.

- CFD calculations, simulation of RTD and comparison with experimental results as a validation of hydrodynamics modelling.

- CFD simulation of the transport of the moments of the age distribution and computation of the degree of mixing.

- 1D modelling of one bed of a SMB process resulting from the previous step. Validation of the 1D model by comparing the obtained results with those obtained from the CFD.

Sections 2 and 3 present the experimental setup and the numerical modelling respectively. The validation of the CFD approach is presented in section 4. Finally in section 5, CFD-based moment calculations are used to reconstruct the RTD, and a 1D model is proposed to represent complex hydrodynamics of adsorption beds.

\section{EXPERIMENTAL SETUP}

The dispersion caused by the different factors introduced above was studied using a cold mock-up representative of a slice of a SMB bed, whose dimensions are shown in Fig. 1. The plates of the distribution system of the SMB are divided into panels that withdraw the flow from the bottom of the packed bed and mix it with an injected feed from the distribution network in order to send it to the packed bed below (see for example the patent US20110303602 A1, Augier and Hotier, 2011).

The experimental setup comprises a packed bed of glass spheres, within which a cylinder or a square prism can be placed as obstacles, with a free flow chamber above the bed and another one below it (see Fig. 2). Above and below the packed bed a tight grid is placed to avoid the movement of the packing due to the motion of the fluid. The inlet pipe ( $R=0.021 \mathrm{~m}$ as for the outlet pipe) that arrives at the top of the mock-up passes in a chamber with three holes ( $r=0.013 \mathrm{~m}$ each) to ensure the flow to be almost two-dimensional. 

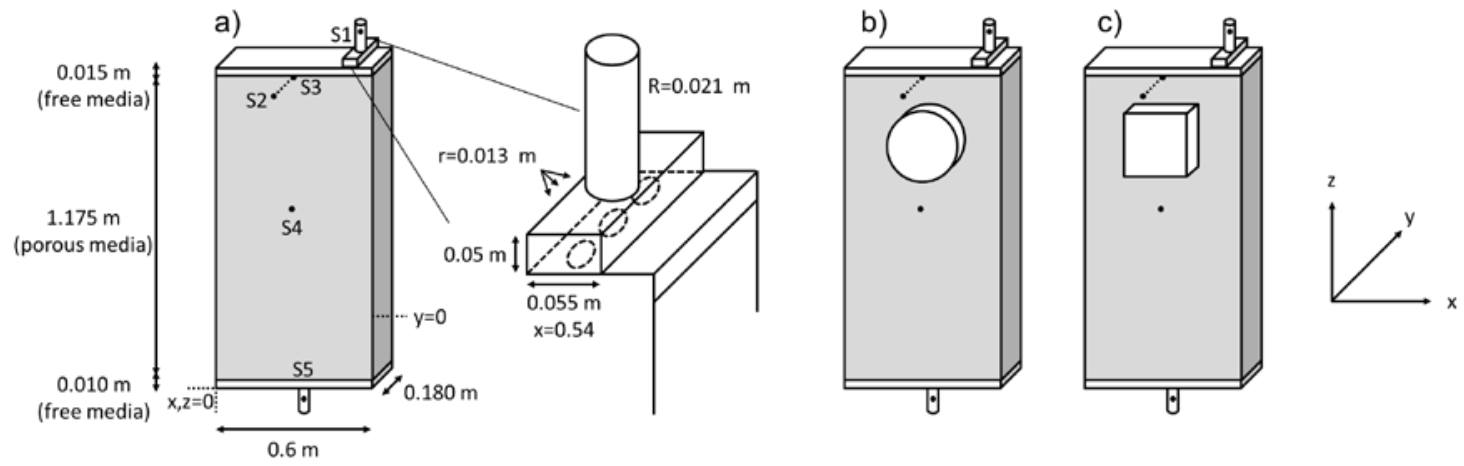

Fig. 2 - Scheme of the experimental setup, respective dimensions and different configurations.

The experimental setup comprises $1.175 \mathrm{~m}$ of height of a packed bed of glass spheres with a $1 \mathrm{~mm}$ diameter and a resulting average porosity of 0.357 (volume void-space divided by the total volume), between two free flow chambers: one above the bed with $0.015 \mathrm{~m}$ and one below it with $0.010 \mathrm{~m}$. The size of glass spheres is chosen to generate a friction in water similar to the friction of xylene at the process temperature $\left(\approx 180^{\circ} \mathrm{C}\right)$ in a fixed bed of $650 \mu \mathrm{m}$ spherical particles of zeolites. Tracer experiments were performed at room temperature by injecting a solution of $50 \mathrm{~g} / \mathrm{L}$ of $\mathrm{KCl}$ in the inlet water stream and by measuring the conductivity with the aid of 5 HEITO-XCP4 sensors of $8 \mathrm{~Hz}$ frequency (with a $1.2 \mathrm{~cm}$ diameter and $20 \mathrm{~cm}$ long). The conductivity sensor S1 placed in the inlet pipe obtains the injection profile of concentration to be used in the CFD simulations. The sensor S5 sensor at the outlet is used to obtain the RTD as shown in Fig. 2 a). The pair of sensors S2 and S3 is placed at $3 \mathrm{~cm}$ down from the top of the packed bed $(x=0.250 \mathrm{y}=0.090$ and $y=0.010 \mathrm{z}=1.155 \mathrm{~m})$ to verify if the flow is two-dimensional. The sensor $S 4(x=0.300 y=0.090 z=0.600 \mathrm{~m})$ is placed below the obstacles to obtain an internal concentration evolution and perceive the distortion caused by the presence of the obstacles. Preliminary tests showed that the response time of the sensors is inferior to $0.125 \mathrm{~s}$.

These tests were performed with a volumetric flow of $6 \mathrm{~m}^{3} / \mathrm{h}$ which corresponds to a superficial fluid velocity of $0.015 \mathrm{~m} / \mathrm{s}$, close to the SMB operating conditions. Afterwards a cylinder $(0.150 \mathrm{~m}$ of radius and $0.175 \mathrm{~m}$ of depth) as shown in Fig. $2 \mathrm{~b}$ ) or a square prism $(0.265 \times 0.265 \times 0.175 \mathrm{~m})$, in Fig. $2 \mathrm{c}$ ) were introduced within the packed bed (both centred in $x=0.300 \mathrm{z}=0.875 \mathrm{~m}$ ). For each configuration the tests were repeated so that the dispersion provoked by the obstacle could be measured. The experimental results were then compared to those obtained through CFD (section 4), in order to validate the model used in the simulation of the flow in the free flow turbulent regions and the porous media.

\section{THEORETICAL BACKGROUND}

\subsection{Residence Time Distribution and Degree of Mixing}

The RTD (Danckwerts, 1953) is widely used to describe the macromixing and hydrodynamics of industrial equipment. This method consists in the measurement of the outlet concentration over time, after an inert tracer injection. The exit age distribution can be obtained through the velocity weighted concentration of tracer in the outlet of the vessel:

$E(t)=\frac{\int_{0}^{L_{e}} C_{e}(x, t) \cdot v_{e}(x) d x}{\overline{v_{e}} \int_{0}^{L_{e}} \int_{0}^{\infty} C_{e}(x, t) d t d x}$

where the subscript $e$ indicates that the concentration and the fluid velocity are measured at the exit. Since the modelling of the experimental setup is performed in two-dimensions, the projection of the outlet cross-section results in a line of length $L_{e}$. The function $E(t)$ gives the probability of a fluid element to leave the vessel, at a given time $t$ after its introduction in S1 at time $t_{0}$. Its integral is equal to unity. The average fluid velocity in the exit is then given by: 
$\overline{v_{e}}=\frac{1}{L_{e}} \int_{0}^{L_{e}} v_{e}(x) d x$

To perform a quantitative analysis of probability density functions (PDF), it is usual to rely on their moments. The $\mathrm{n}^{\text {th }}$ raw and the $\mathrm{n}^{\text {th }}$ central moments can be calculated according to:

$m_{n}=\int_{0}^{\infty} t^{n} \cdot E(t) d t$

$\mu_{n}=\int_{0}^{\infty}\left(t-m_{1}\right)^{n} \cdot E(t) d t$

The first moment of the RTD is the mean of the distribution $\left(m_{1}=\mu_{1}\right)$, which is equal to the average residence time of a vessel, given by the ratio between the volume of the vessel and the inlet volumetric flow in the absence of dead volumes. The second central moment of the RTD is the variance $\left(\sigma^{2}\right)$. Its positive square root is the standard deviation. In the case of an ideal plug flow, the $\sigma^{2}$ of the RTD is equal to 0 . The fluid elementary volumes that enter together in the vessel leave the system at the same time. The third central moment is the skewness. It measures the asymmetry of a PDF. By measuring the first two moments of the RTD it is possible to obtain the mean age and the dispersion in the outlet of the studied vessel. This is sufficient to fit simple flow models as DPF or CSTR cascade.

The RTD is useful for characterizing the macromixing of a given system, although the internal system information remains inaccessible. If we consider a system of two consecutive reactors, for example one CSTR and one Plug Flow Reactor (PFR), the resulting RTD is the same regardless the order in which the two reactors are placed. However, as Danckwerts (1953) pointed out, in the presence of non-linear phenomena, for example a chemical reaction with second order kinetics, the two systems (CSTR+PFR and PFR+CSTR) will produce different performances. To explain this, Danckwerts (1958) and Zwietering (1959) formulated a criterion: the degree of mixing that quantifies the quality of the mixing of a given vessel. The degree of mixing can vary between 0 (perfectly mixed system, such as an ideal CSTR) and 1 (completely segregated system, PFR). The degree of mixing is defined as the variance of the mean age of the fluid elements averaged over all the points of a vessel, divided by the variance of the ages of all the fluid elements within a vessel. For about 50 years the degree of mixing could not be quantified, but Liu (2012) showed that it can be calculated through the volume average of the first two moments of the internal age distribution:

$J=\frac{\overline{m_{1, V}^{2}}-\bar{m}_{1, V}^{2}}{\bar{m}_{2, V}-\bar{m}_{1, V}^{2}}$

Knowing that at any given point of a vessel there is a distribution of the age of the fluid passing by, Liu and Tilton (2010), following the work of Spalding (1958) and Sandberg (1981), showed that its raw moments are transported by diffusion and convection and can be obtained through steady state CFD simulations:

$\nabla \cdot\left(\vec{u} m_{n}-D \nabla m_{n}\right)=n \cdot m_{n-1}$

The coefficient $D$ comprises all the dispersive phenomena present in the vessel (such as turbulent mixing or mechanical dispersion). The source term on the right-hand side is the product between the order of the moment transported $(n)$ and the $(n-1)^{\text {th }}$ moment (i.e. $m_{n-1}$ ), i.e. the source term is equal to 1 when transporting the first moment, and equal to $2 m_{1}$ when transporting the second raw moment. With this, it is possible to obtain the first two moments of the age distribution, in every point of a vessel, through steady state simulations that are much cheaper, in terms of computational time, than dynamic simulations of tracer injections. Baléo and Le Cloirec (2000) and Jongen (2004) simulated the 
transport of the first moment of the age distribution through the equation deduced by Spalding (1958), although the transport of moments of order higher than one was not possible. For a system that comprises a cascade of $N$ CSTR, that share the same residence time, the degree of mixing of the system can be obtained through the number of enchained CSTR (appendix A):

$J=\frac{N-1}{N+5}$

A classic approach based on the first two moments of a given RTD may lead to several candidate models. The degree of mixing $J$ may be useful to differentiate them. Among them, the closest degree of mixing may be a guarantee of an accurate hydrodynamic representation.

\subsection{Hydrodynamic modelling}

As the experimental setup and the adsorption beds exhibit a porous media between two free flow areas, the CFD model has to solve both flow configurations, leading to 2 distinct set of equations. The simulations were performed with COMSOL 4.3b software.

\subsubsection{Free flow}

In the free flow volumes adjacent to the porous media, the flow is described using the ReynoldsAveraged Navier-Stokes equations (RANS) for a Newtonian fluid and an incompressible and steady flow:

$\rho \vec{u} \cdot \nabla \vec{u}+\nabla \cdot \frac{2}{3} \rho k=-\nabla p+\nabla \cdot\left(\mu+\mu_{T}\right)\left(\nabla \vec{u}+(\nabla \vec{u})^{T}\right)$

$\rho \cdot \nabla \overrightarrow{\vec{u}}=0$

Here, $\vec{u}$ is the averaged velocity field and $k$ the turbulent kinetic energy. The turbulent dynamic viscosity $\mu_{T}$ is modelled by the standard $k-\varepsilon$ turbulence model for a steady and incompressible flow (Jones and Launder, 1972):

$\mu_{T}=\rho \cdot C_{\mu} \frac{k^{2}}{\varepsilon_{T}}$

The turbulent kinetic energy $k$ and its dissipation rate $\varepsilon_{T}$ are transported according to:

$\rho \cdot \vec{u} \cdot \nabla k=\nabla \cdot\left(\left(\mu+\frac{\mu_{T}}{\sigma_{k}}\right) \cdot \nabla k\right)+P_{k}-\rho \cdot \varepsilon_{T}$

$\rho \cdot \vec{u} \cdot \nabla \varepsilon_{T}=\nabla \cdot\left(\left(\mu+\frac{\mu_{T}}{\sigma_{\varepsilon}}\right) \cdot \nabla \varepsilon_{T}\right)+C_{\varepsilon 1} \frac{\varepsilon_{T}}{k} P_{k}-C_{\varepsilon 2} \cdot \rho \frac{\varepsilon_{T}{ }^{2}}{k}$

$C_{\mu}, \sigma_{k}, \sigma_{\varepsilon}, C_{\varepsilon 1}$ and $C_{\varepsilon 2}$ are $k-\varepsilon$ model constants determined from experimental data (Launder and Spalding, 1974).

The production term $P_{k}$ is calculated by:

$P_{k}=\mu_{T} \cdot\left(\nabla \vec{u}:\left(\nabla \vec{u}+(\nabla \vec{u})^{T}\right)-\frac{2}{3} \cdot(\nabla \cdot \vec{u})^{2}\right)-\frac{2}{3} \rho \cdot \nabla k \cdot \vec{u}$

In order to describe the mixing of a scalar due to eddy motion, a turbulent diffusivity coefficient must be calculated through the turbulent viscosity and by imposing a constant turbulent Schmidt number $S c_{T}$. Such diffusivity coefficient must be added to the molecular diffusion, in the free flow volumes, to describe properly the mixing in the turbulent regions. Koeltzsch (2000) reviewed several experimental investigations and stated that $0.5<S c_{T}<0.9$, Flesch et al. (2002) pointed out that $0.18<S c_{T}<1.34$ and Combest et al. refer that $S c_{T}=0.7$. The latter will be used in this work. This model relies on several 
assumptions. The most important are that the Reynolds number is sufficiently high and that the turbulence viscosity is isotropic. The flow is assumed to be stationary in both porous and free flow.

The tracer molecules are transported by diffusion and convection. The transport equation in the free flow is:

$\frac{\partial C}{\partial t}=\nabla \cdot\left(\left(D_{M}+D_{T}\right) \cdot \nabla C-\vec{u} C\right)$

The global dispersion coefficient results from the sum of the molecular diffusion $\left(D_{M}\right)$ and the turbulent diffusivity $\left(D_{T}\right)$. Both are assumed isotropic.

\subsubsection{Porous media}

The model developed describes hydrodynamics and scalar transport at a scale larger than the packing particles but much smaller than the experimental setup. This is acceptable due to the high ratio between the bed dimensions and the particle diameter. The contact between the fluid and the particles is not simulated in the CFD, but modelled through a diffusive term in the momentum and transport balance equations (Eqs. 15 and 19). To do this, the Brinkman-Forchheimer model (Vafai and Tien, 1982) is used:

$\frac{\rho}{\varepsilon}\left((\vec{u} \cdot \nabla) \frac{\vec{u}}{\varepsilon}\right)=\nabla \cdot\left[-p+\frac{\mu_{e}}{\varepsilon}\left(\nabla \vec{u}+(\nabla \vec{u})^{T}\right)-\frac{2 \mu}{3 \varepsilon}(\nabla \cdot \vec{u})\right]-\left(\rho \cdot \beta \cdot|\vec{u}|+\frac{\mu}{K}\right) \vec{u}$
$\rho \cdot \nabla \vec{u}=0$

Here, $\vec{u}$ is the superficial fluid velocity. This model results from a modification of the laminar NavierStokes equations and takes into account the diffusion of the momentum due to viscosity effects through the Brinkman term $\left(\mu_{e} \cdot \nabla^{2} \vec{u} / \varepsilon\right)$ and the inertial effects caused by friction between the fluid and the particles with the use of the Forchheimer term $(\rho \cdot \beta \cdot|\vec{u}| \cdot \vec{u})$. The validity of this model has been discussed by Nield (1991), Vafai and Kim (1995), Augier et al. (2008), and it has been rather successfully compared to experimental results by Benyahia (2004) and Chan et al. (2000), although its applicability to interfaces between free turbulent and laminar porous media regions was not verified. The effective dynamic viscosity $\mu_{e}$ has been widely discussed. Some authors propose its calculation as a function of the particle Reynolds number, $\mu_{e}=\mu_{T}\left(R e_{p}\right)$ (Guo et al., 2006) while others defend that for low particle Reynolds numbers it can be assumed equal to the molecular viscosity, $\mu_{e}=\mu$ (Chan et al., 2000). In this work $\mu_{e}=\mu$ will be assumed. For a fixed bed of spheres, the bed permeability $K$ and the non-Darcy coefficient can be calculated through Ergun's law (1952):

$$
\begin{aligned}
K & =\frac{\varepsilon^{3} d_{p}^{2}}{150(1-\varepsilon)^{2}} \\
\beta & =1.75 \frac{1-\varepsilon}{\varepsilon^{3} d_{p}}
\end{aligned}
$$

This law was developed for one-dimensional flows within isotropic media, but Zeng and Grigg (2006) showed that its application is acceptable when the permeability term of Eq. (15) is more important than the Forchheimer term, which is fulfilled in this case. The tracer molecules are transported throughout the inter-particle void volume according to:

$\varepsilon \frac{\partial C}{\partial t}=\nabla \cdot\left(\varepsilon\left(D_{M}+D_{P}\right) \cdot \nabla C-\vec{u} C\right)$

Within the porous media the global dispersion coefficient results from the sum of the molecular diffusion and the mechanical dispersion due to the packing $\left(D_{P}\right)$. While the molecular diffusion is isotropic, the mechanical dispersion is described by a diagonal tensor comprising the transversal $\left(D_{t r}\right)$ and axial dispersion $\left(D_{a x}\right)$ coefficients, relatively to the flow direction. These two coefficients are calculated by imposing two constant Peclet numbers based on the particle diameter, one transversal $\left(P e_{t r}\right)$ and one axial $\left(P e_{a x}\right)$, that quantify the ratio between convective and diffusive transport. The transversal and axial Peclet numbers based on the particle diameter are assumed to be 11 and 2 respectively, following the results obtained by Foumeny et al. (1992). Delgado (2006) showed that 
these values are considered to be asymptotic for liquid flows with particle Reynolds numbers greater than 20. In order to couple the two free flow regions with the porous media, the boundary conditions used made that the pressure and the horizontal and the vertical components of the fluid velocity are the same on both sides of the interfaces, for each mesh element.

\section{VALIDATION OF THE CFD MODEL}

The RTD obtained experimentally exhibits long tails and some residual noise associated to the conductivity sensors, as shown in Fig. 3 a).
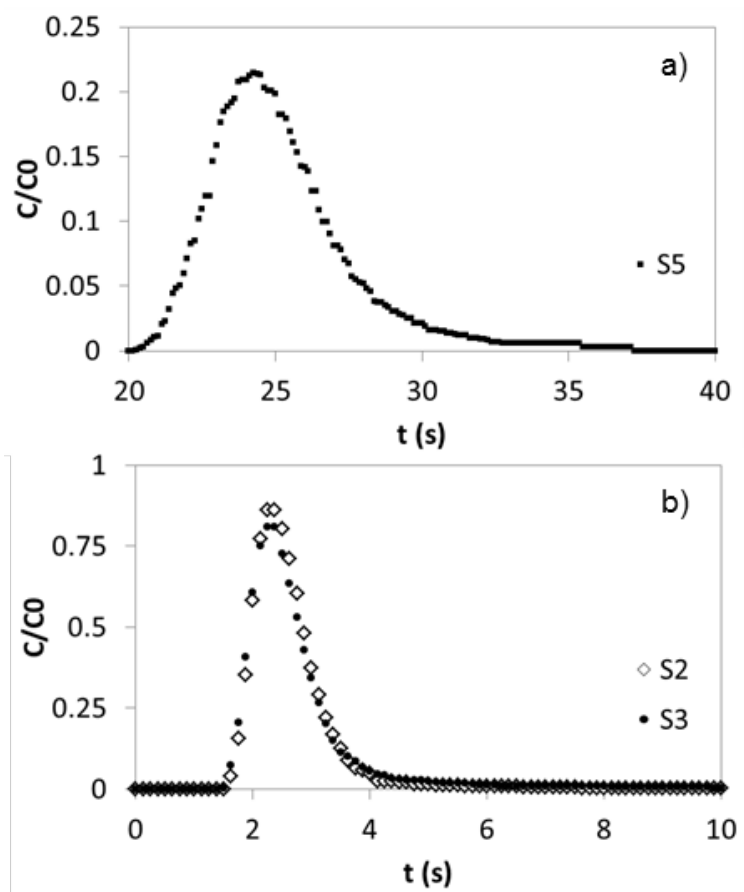

Fig. 3 - Experimental RTD obtained in the absence of obstacle (a) and concentration curves of the sensors S2 and S3 (b).

The signal noise in the tail of the RTD induces considerable errors in the measurement of the moments of order higher than one. Any signal post treatment or arbitrary choice of the final measuring time may strongly influence high order moments. Thus, the validity of the model will be studied through the visual comparison between the experimental and numerical RTD, rather than comparing its moments.

The concentration curves obtained with the sensors S2 and S3 shown on Fig. 3 b) indicate that the flow inside the mock-up can be considered as 2D, easing its CFD modelling. The parameters used in the 2D CFD simulations are shown in Table 1.

Table 1: Simulation parameters.

\begin{tabular}{ccc}
\hline Parameter & Value & Units \\
\hline$\varepsilon$ & 0.357 & - \\
$\rho$ & 1000 & $\mathrm{~kg} / \mathrm{m}^{3}$ \\
$\mu$ & 0.001 & Pa.s \\
$D_{M}$ & $1.00 \times 10^{-9}$ & $\mathrm{~m}^{2} / \mathrm{s}$ \\
$d_{p}$ & $1.00 \times 10^{-3}$ & $\mathrm{~m}$ \\
$P e_{a x}$ & 2 & - \\
$P e_{t r}$ & 11 & - \\
$\mathrm{Sc}_{T}$ & 0.7 & - \\
$S c_{T}$ & &
\end{tabular}




$u_{\text {in }} \quad 1.132 \quad \mathrm{~m} / \mathrm{s}$

The near-wall porosity profiles used by Augier e al. (2008) were tested but the results obtained were comparable to those with constant bed porosity. The dimensionless numbers used were chosen from the bibliography described above, and no parameter fitting was performed. The no slip condition was applied on the walls and the mesh is shown in Fig. 4. The mesh elements are around 100 times smaller than the obstacles.

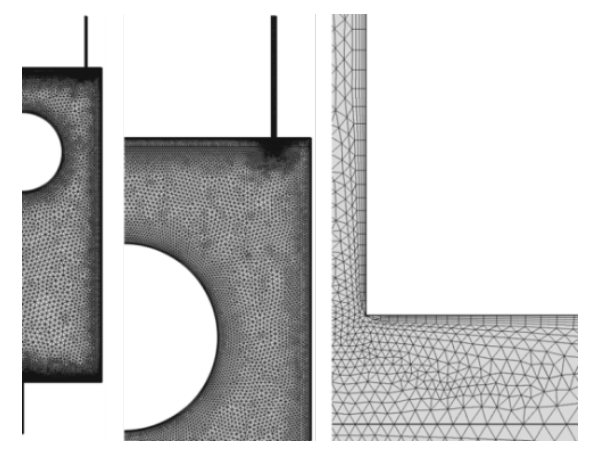

Fig. 4 - Adaptive mesh used in the CFD simulations.

The mean age, the variance and the skewness obtained at the outlet of the setup and the degree of mixing are shown in Table 2. The experimental and numerical RTD are compared in Fig. 5. All the curves are centred on their first moments.

Table 2: First three central moments of the RTD and degree of mixing for the two geometrical configurations.

\begin{tabular}{ccccccccc}
\cline { 3 - 9 } & & $\mu_{1}(\mathrm{~s})$ & $\sigma^{2}\left(\mathrm{~s}^{2}\right)$ & $\mu_{3}\left(\mathrm{~s}^{3}\right)$ & $J(-)$ & $N(-)$ & $(N-1) /(N+5)(-)$ & $2 N \tau^{3}\left(\mathrm{~s}^{3}\right)$ \\
\hline \multirow{2}{*}{ No obstacle } & Exp. & 28.8 & 3.4 & 3.2 & - & - & - & - \\
& CFD & 28.8 & 4.1 & 14.5 & 0.990 & 204 & 0.971 & 1.1 \\
\hline \multirow{2}{*}{ Cylinder } & Exp. & 26.1 & 4.9 & 10.0 & - & - & - & - \\
& CFD & 26.1 & 6.9 & 25.9 & 0.980 & 99 & 0.942 & 3.6 \\
\hline \multirow{2}{*}{ Square Prism } & Exp. & 26.1 & 5.4 & 10.9 & - & - & - & - \\
& CFD & 26.1 & 14.5 & 109.8 & 0.963 & 47 & 0.885 & 16.1 \\
\hline
\end{tabular}

The errors due to integration of the experimental RTD are visible when calculating the second moment in the square prism configuration and for the third moment obtained for every geometry. From the CFD results it can be concluded that adding the cylindrical obstacle increases the variance by about $70 \%$ and the skewness by $80 \%$. The square prism has a more important impact, increasing the variance by a factor of 2.6 and the skewness by 6.7. Since the discrepancies between the moments obtained experimentally and numerically are evident, the validity of the model will be evaluated by visual comparison between the obtained RTD. 


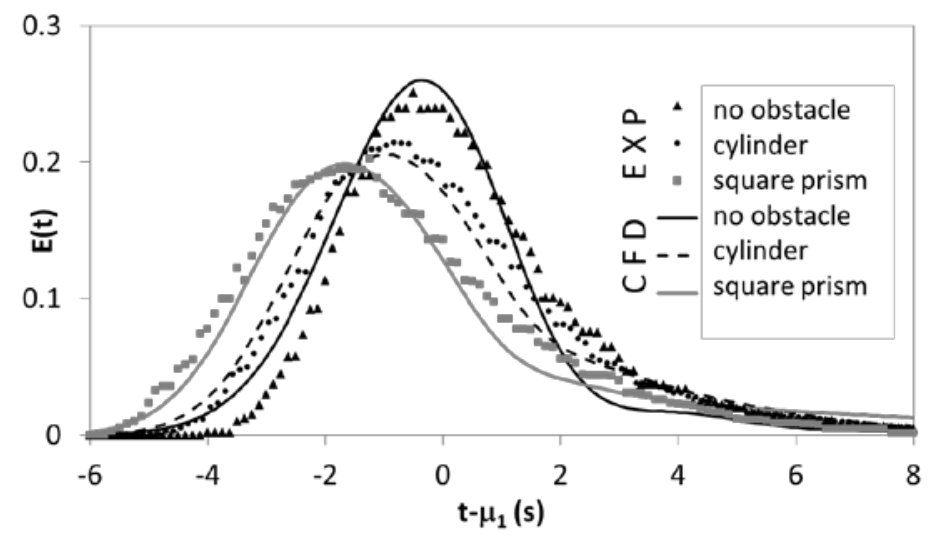

Fig. 5 - Experimental and numerical RTD obtained for the three configurations studied.

A rather good agreement between CFD and experimental values is found as shown in Fig. 5. The CFD model is capable of reproducing the RTD of the experimental setup for the three geometrical configurations: absence of obstacles, cylinder or square prism are placed within the porous media.

Through the transport of the raw moments of the age distribution, it is possible to obtain a mapping of the moments, as shown in Fig. 6 a) and b) for the mean age.

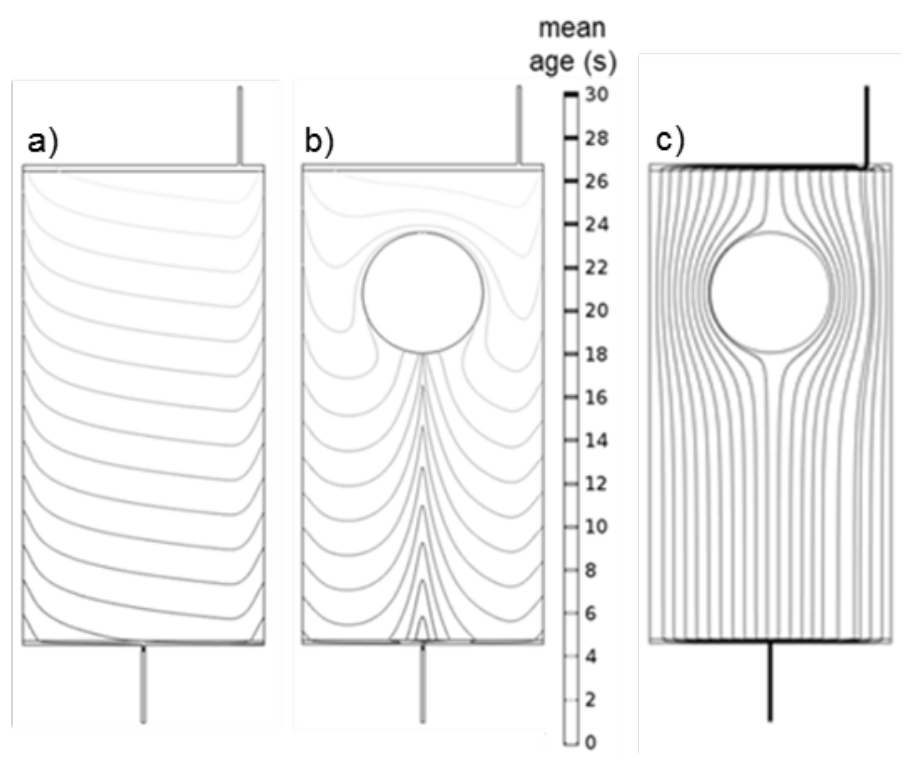

Fig. 6 - Spatial distribution of the mean age without (a) and with a cylindrical obstacle (b) and streamlines of the experimental setup with a cylindrical obstacle (c).

From Fig. 6 a), the fronts of mean age (and consequently the front of concentration, when simulating the tracer injection) advances obliquely since the inlet is positioned on the right side of the mock-up. This explains the tail of the RTD. In the presence of a cylinder within the porous media, the front of ages is severely disturbed. A delayed region is created below the obstacle. Also, the injected elements of fluid arrive quicker on the right-hand side of the obstacle (closer to the inlet) creating a small bypass effect. The same behaviour was found when the square prism was placed in the porous media.

As the degree of mixing depends on the moments of the age distribution inside the vessel, the model developed must be capable of reproducing the concentration curves of tracer in every internal point of the vessel. As it is impossible to verify this for every vessel point, the tracer concentration was measured below the obstacles which is considered to be the most critical point for the numerical model, since this is where the two concentration fronts that are split above the obstacle will meet. The curves obtained are shown in Fig. 7. 


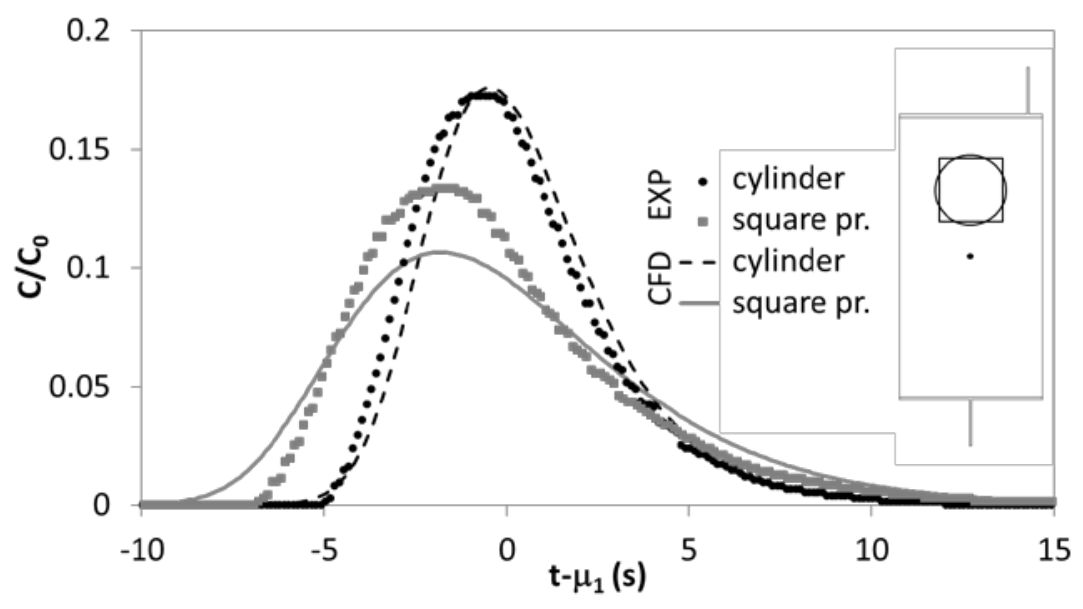

Fig. 7 - Experimental and numerical concentration curves obtained for both obstacles and the position of its measurement.

The CFD model fits well the curves obtained experimentally, despite the slight overestimation of the dispersion $(\approx+9 \%)$ below the square prism. However, the agreement is considered acceptable. As a consequence, in the following, CFD is considered as a reference for the development of the 1D model.

\section{5 "STATIONARY RTD" AND DEVELOPMENT OF A 1D MODEL}

To obtain the RTD of an adsorber through CFD, each simulation can take up to 24 hours to converge on recent scientific computers (4 cores $2.8 \mathrm{GHz}, 6 \mathrm{Go}$ RAM), while the simulation of multicomponent adsorption breakthrough can increase up to 72 hours. In order to obtain stable concentration fronts inside the SMB all the beds need to be simulated through about one hundred cycles. This turns the use of CFD prohibitive. Thus, it is of the highest interest to develop a 1D hydrodynamic model capable of giving an accurate representation of the flow inside a fixed bed adsorber.

The first simple model considered is a cascade of $N$ CSTR of equal residence time $\tau$. $N$ and $\tau$ are fitted to adjust $\mu_{1}$ and $\sigma^{2}$ to CFD calculation results, and associated degree of mixing $(J)$ and skewness $\left(\mu_{3}=2 N \tau^{3}\right)$ are also reported in Table 2. One advantage of the NCSTR model is the analytical calculation of $J$ (Eq.7). The values of the degree of mixing obtained for the three configurations are close to an ideal PFR (Table 2), in spite of all the internal dispersion due to the free flow chambers, the obstacles and the packed bed. The degree of mixing of a cascade of CSTR given by Eq. (7) is lower than those obtained through CFD, for the three geometries, reaching a deviation of $8 \%$ when in the presence of the square prism. Such deviations can induce some inaccuracies in the impact of hydrodynamics on non-linear phenomena if the proposed 1D model is used. Regarding the skewness of the same 1D models, the differences are more important, for the square prism configuration. The 1D model presents a skewness of $16 s^{3}$ much smaller than the CFD $\left(110 s^{3}\right)$ and for the three configurations the relative error is around $-88 \%$. Such skewness deficit results in RTD with much smaller tails leaning to the right, than those obtained through CFD. These results show that the NCSTR model is not relevant in the present cases. Besides, it is known that DPF and NCSTR models behave similarly when $N$ increases. As $N$ is really high $(47<N<204)$ in the studied configurations, one may conclude that DPF models are also irrelevant and may lead to strong errors on degree of mixing and skewness.

More complex 1D models have to be developed, using available information on spatial moment distributions inside the bed. By looking at Fig. 6 b), one can perceive that important differences of mean age distribution are present in the transversal direction. This shows that a major contribution to the age distribution is generated by the heterogeneities of streamlines in the transversal direction (Fig. 
$6 \mathrm{c})$. The different lengths are caused by the distribution and withdrawal devices above and below the porous media, and by the presence of the obstacle. As the separating area is the porous media, a focus is proposed for now without considering the free flow zones before and after the fixed bed. This fictitious case allows us to study the isolated selective zone in a first time, which is not possible experimentally. In a second time, the non-selective free zones are added to the previous case. In both cases a 1D model based on the transport of internal age distribution is proposed.

\section{a) Selective porous media without free flow zones}

As the degree of mixing of the porous media is close to a completely segregated system, it is proposed to represent the bed as a set of parallel NCSTR models (PNCSTR) of different residence times and variances, as shown in Fig. 8 a).

a)

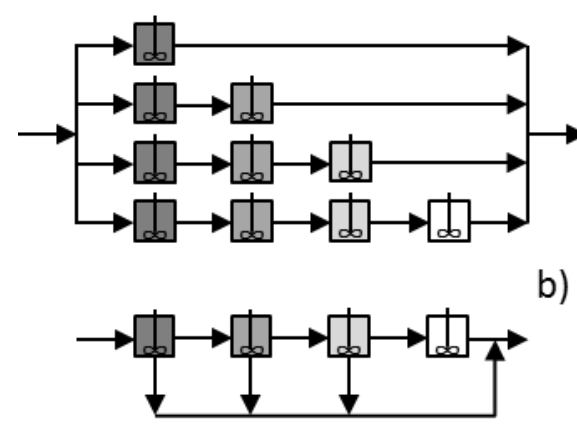

Fig. 8 - PNCSTR model (a) and equivalent multiple exit NCSTR (MENCSTR) model (b).

To illustrate this, a fictitious geometry is proposed where the two free flow zones are removed from the experimental setup and only the packed bed with the cylindrical obstacle placed inside are studied. The homogeneous inlet superficial velocity is $0.015 \mathrm{~m} / \mathrm{s}$. A three-step simulation, that we call "Stationary RTD", is performed to adjust the PNCSTR model to the CFD results. This consists in:

- Simulation of the flow within the studied geometry.

- Transport of the first two moments of the distribution of ages of the fluid.

- Use of these two moments at the outlet of the geometry and solving the analytical equation of a NCSTR model for each mesh element of the outlet through Eqs. (20), (21) and (22) (Schweich, 2001).

$E(t)=\left(\frac{N}{\tau}\right)^{N} \frac{t^{N-1} \exp (-N t / \tau)}{(N-1) !}$

Where $\tau$ and $N$ are given by:

$\tau=m_{1} / N$
$N=\frac{m_{1}^{2}}{m_{2}-m_{1}^{2}}$

Eq. (20) is solved once for each mesh element and in order to obtain the stationary RTD, all the $\omega_{T}$ solutions of $E(t)$, where $\omega_{T}$ is the number of mesh elements at the outlet, must be weighted by the fluid velocity in the mesh element and by the mesh element size $l$, as shown in Eq. (23): 
$\operatorname{StatRTD}(t)=\frac{\sum_{i=1}^{\omega_{T}} E(t) \cdot v_{e} \cdot l}{\sum_{i=1}^{\omega_{T}} v_{e} \cdot l}$

The stationary RTD ( $\omega_{T}=200$ mesh elements) is compared to the RTD calculated by CFD and by a NCSTR model with the fitting of the first two moments of the RTD in Fig. 9.

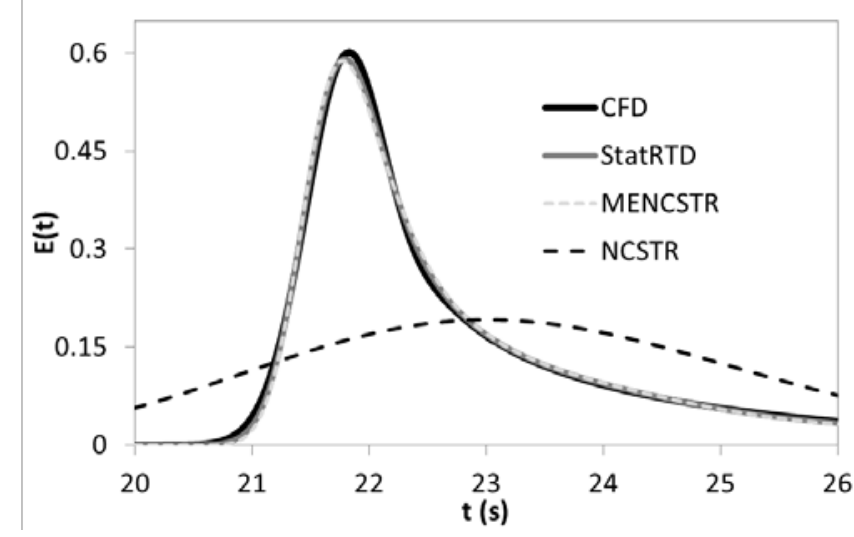

Fig. 9 - RTD comparison between the different models.

The Stationary RTD obtained matches the RTD obtained through CFD in a very satisfactory way while the NCSTR model is incapable of reproducing asymmetric RTD. The Stationary RTD, in this case, takes few minutes to converge, while the CFD simulation of the RTD can take up to a day.

In order to approach the geometrical configuration of a SMB packed bed, two types of obstacles are studied (pipe and beam) in a larger bed (Fig. 10 a). The inlet superficial velocity remains $0.015 \mathrm{~m} / \mathrm{s}$.
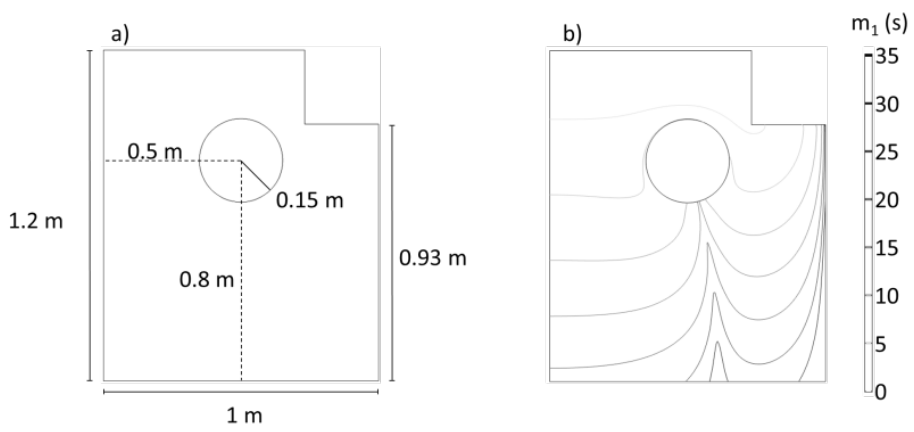

Fig. 10 - Geometry and dimensions of the fictitious geometry (a) and the obtained curves of $m_{1}$ (b).

It was not possible to perform an experimental study comprising the two obstacles simultaneously because it would exceed the pressure drop specification of the mock-up.

The histogram of the weighted $m_{1}$ in the outlet of the packed bed, as well as the resulting Stationary RTD ( $\omega_{T}=200$ mesh elements), are shown in Fig. 11. The Stationary RTD is compared to the RTD calculated by CFD, by a NCSTR with the fitting of the first two moments of the age distribution and by the equivalent PNCSTR model. 

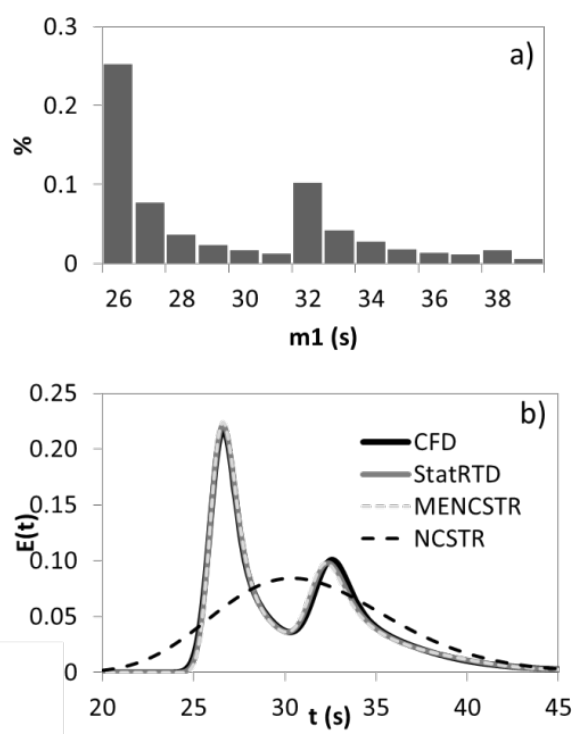

Fig. 11 - Histogram of the weighted $m_{1}$ obtained at the outlet of the bed (a) and RTD comparison between the different models (b).

The NCSTR model is clearly not suitable for the present application as it generates an unimodal and symmetric RTD. The Stationary RTD overlaps almost perfectly the RTD generated by CFD, and they are comparable to the histogram of the first moment in the outlet of the vessel. In this case, it is possible to state that the RTD is given by the distribution of the first moment in the outlet and the associated dispersion. While the simulation of the tracer progression throughout the packed bed takes about a day to converge, the transport of the moments of the distribution of ages of fluid elements takes a couple of minutes to converge and the solution of Eq. (20) is instantaneous. This makes feasible and fast the study of different geometrical bed configurations. The validity of this approach relies on three major assumptions:

- The concentration curve obtained in each mesh element must have a single local maximum, else it cannot be replicated by a NCSTR model.

- The asymmetry of $m_{1}$ in the outlet (Fig. 11 a) must be dominant compared to the asymmetry of the concentration curves obtained locally, due to the incapability of the NCSTR model to reproduce a skewed RTD.

- The transversal diffusion is not important, so that the system can be represented by a PNCSTR model, although a sensitivity analysis was performed, and even when transversal diffusion is 100 times stronger than in this case, this approach remains valid.

In this case, the bimodality of the RTD is caused by the split of the concentration front due to the presence of the pipe. The two concentration fronts arrive at the outlet of the bed at different times as shown in Fig. 10 b).

200 outlet mesh elements were used to reproduce the RTD of a singled bed, therefore the Stationary RTD shown above is equivalent to a set of 200 NCSTR models. In order to study the SMB, cyclic simulations of multicomponent adsorption must be carried on throughout a multitude of beds. Therefore, it is interesting to reduce the number of NCSTR per bed in order to reduce the computation time. For this, an additional assumption is made. If we consider that each NCSTR model can be built as a prolongation of the shorter one, the longest NCSTR model might result in a combination of all the others, as shown in Fig. 8.

A multiple exit NCSTR (MENCSTR) model can be built by identifying the shortest cascade (with a residence time $\tau_{1}$ ) of the PNCSTR model and adding to it a number of CSTR with a residence time $\tau_{2}$ in order to adjust the variation of $m_{1}\left(\Delta m_{1}\right)$ and $\sigma^{2}\left(\Delta \sigma^{2}\right)$ between the shortest and the second shortest cascades. While the first is always positive, the second, $\Delta \sigma^{2}$, can be negative, i.e. a given streamline can be less dispersed that a shorter streamline. Since it is not possible to add negative dispersion, 
each time $\Delta \sigma^{2}$ is null or negative, a high number of CSTR is added in order to avoid the overestimation of the dispersion.

Zwietering (1959) introduced the multi-exit plug flow reactor as an example of a completely segregated system. This is convenient in our case due to the high degree of mixing of the adsorber. However, in our case, the side exits model has an associated dispersion due to the residence time and number of CSTR that vary between two consecutive side exits, in order to adjust the variation of $\sigma^{2}$ between two consecutive NCSTR models (when ordered by length). With this simplification, it is possible to obtain a resulting RTD that matches perfectly those shown in Fig. $11 \mathrm{~b}$ ). This also decreases considerably the CPU times compared to CFD or PNCSTR models.

\section{b) Selective Porous media surrounded by free flow zones}

In order to describe the studied geometry hydrodynamics, it is required to dissociate the porous media, responsible for the adsorptive separation, from the free flow zones. The two free flow zones are therefore integrated in the CFD geometry. In order to dissociate the three zones, the transport of the first and second moments is studied separately by solving the source term of Eq. (6) in one region at a time. Then, we discretize the fluid in $n$ vertical elements (11 in Fig. 12 a) and assume that the $n^{\text {th }}$ fluid element at the entrance of the porous media corresponds to the same $n^{\text {th }}$ element in the exit. By doing this, it is possible to link the moments obtained for each of the three regions. The moments obtained in the porous media are used to define a side exit model like the one in Fig. $8 \mathrm{~b}$ ) where the adsorption will take place. Then, each exit is linked to a second NCSTR model at different points (Fig. $12 \mathrm{c}$ ). $\tau$ and $N$ between the entrances are adjusted so that each side exit experiences the same delay $\left(m_{1}\right)$ and dispersion $\left(m_{2}-m_{1}^{2}\right)$ that the corresponding fluid element did in the CFD transport of the moments (above the bed (subscript A) and below (subscript B) the porous media). The free flow zones consist in continuous mixing apparatus, since the adsorptive separation does not take place there, thus their degree of mixing is unimportant. If we consider that the first free flow zone consists in a mixing apparatus due to the heterogeneity of the horizontal profile of $m_{1}$ generated in the interface free/porous media, rather than by actual local $\sigma^{2}$, it can be modelled as later stage mixing. In this case, this approximation is valid since the variance of the profile of $m_{1}$ weighted by the local velocity is $0.44 \mathrm{~s}^{2}$, much higher than the velocity averaged $\sigma^{2} 0.03 \mathrm{~s}^{2}$.

Since the internal mixing of the adsorption bed is low (degree of mixing of 0.99), it can be described as a system of parallel cascades of CSTR, as described previously (see Fig. 8). Since the free flow zones are non-selective, and that they generate relatively low mixing but high delays, they are combined and modelled after the adsorption bed (see Fig. 12 b). 
a)

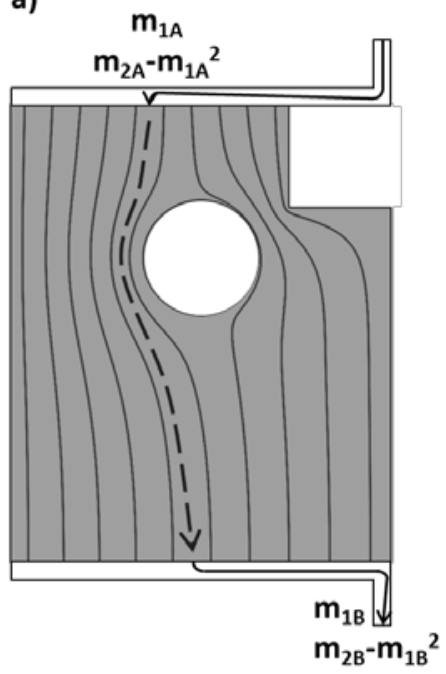

b)

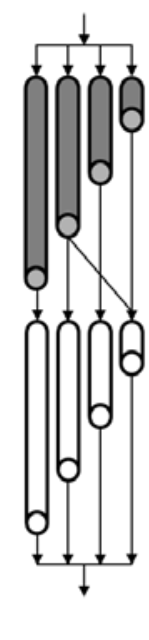

c)

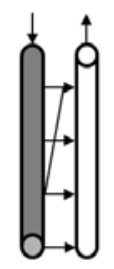

adsorption

no adsorption

Fig. 12 - Scheme of the modelling proposed (a), equivalent system of parallel NCSTR models (b) and resulting multiple entrances and multiple exits CSTR cascades (2MENCSTR) (c).

Fig. 12 illustrates the modelling of the non-selective zones that will be explained with more detail in appendix B. The complete system, composed by the two free flow zones and the adsorption bed, can be described by a first system of parallel NCSTR models where adsorption takes place, linked to a second set of parallel where adsorption does not take place (equivalent to the free flow zones). These two systems of parallel NCSTR can be simplified resulting in a first MENCSTR where adsorption takes place that is connected with side streams to a second NCSTR where adsorption does not take place, as described on Fig. $12 \mathrm{c}$ ). The connections and parameters of this 1D model are chosen to fit with the CFD. This modelling approach was verified for several bed geometries resulting in good agreement between the CFD and the 1D model. Fig. 13 shows the RTD obtained through CFD when the two free flow zones were added to the packed bed and its comparison with the RTD of the NCSTR model and the with the multiple exits and entrances model (2MENCSTR).

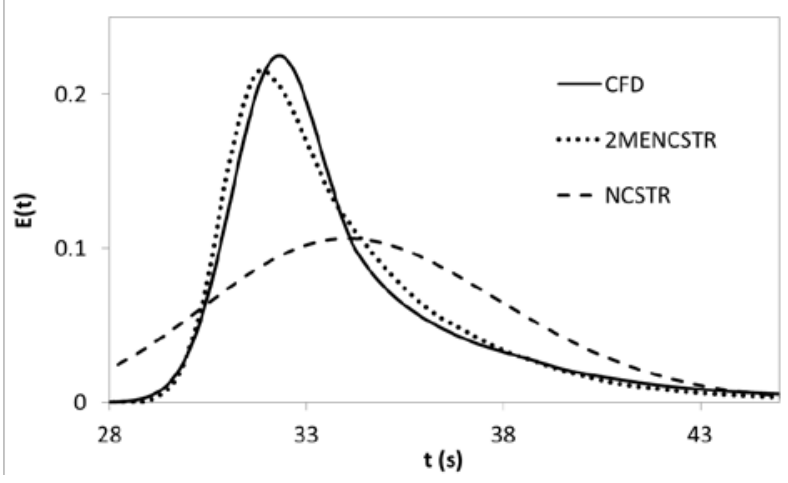

Fig. 13 - RTD obtained with the CFD and the different models studied, for the complete geometry.

The NCSTR model is unable to reproduce the RTD amplitude and asymmetry, even after adjusting the first two moments. On the other, hand RTD of the 2MENCSTR model is much closer to the one obtained by CFD (see Fig. 13). The 2MENCSTR RTD does not match perfectly the RTD obtained by CFD. Since the RTD of the adsorption bed without the free flow zone using the 1D MENCSTR model is very close to CFD RTD (see Fig. 9), we can conclude that the model of the free flow zones as a unique NCSTR system introduces a small error. Nevertheless with this model, it is possible to obtain results very close to the ones obtained by CFD of multicomponent adsorption within few seconds, instead of 72 hours. This makes feasible the study of several parameters on the process performance with a fine hydrodynamic description. 
A MENCSTR model could be fitted to the RTD of the complete geometry (porous media and free flow), but it would not be possible to separate the packed bed, where adsorption will be coupled with in further studies, from the free flow regions. However, such distinction between zones is possible when using a 2MENCSTR model, by coupling adsorption the first cascade and leaving the second one to be non-selective.

Since the development of this model respects the global degree of mixing in addition to the RTD, a good behaviour of the model is expected once coupled with nonlinear adsorption isotherms. Since the calculation of hydrodynamics is very quick (few seconds), the developed approach will be used in the future for simulation of industrial SMB processes.

\section{CONCLUSIONS}

The CFD model developed is capable of reproducing the RTD and the internal concentration curves of the experimental setup without any parameter fitting. The coupling between the BrinkmanForchheimer and the standard $k-\varepsilon$ turbulence model reproduces the flow at the interfaces between a turbulent region and the porous media, with an acceptable accuracy. The dispersion and skewness are well reproduced when comparing CFD results with experimental ones.

The degree of mixing can only be obtained through CFD simulations of the transport of the distribution of ages moments in the fluid elements (Liu, 2012). It shows that the flow is close to a completely segregated system, despite the internal dispersion of the experimental setup, due to the free flow chambers, the packing and the obstacle. The impact of the setup hydrodynamic, which is representative of a SMB bed, on adsorptive separation may not be fairly reproduced by a NCSTR or a DPF model. It is not possible to adjust such models to the setup degree of mixing and its RTD skewness. Thus, through the transport of the first two moments of the age distribution, it is possible to obtain a Stationary RTD, i.e. the RTD can be obtained through the interpretation of these moments in the outlet of the porous media. Moreover the transport of the moments takes few minutes to converge, much less than the CFD simulation of the RTD that can take up to a day. Because of this result, we suggest the use of a PNCSTR model to simulate the flow inside the porous media.

In order to save time when simulating multicomponent adsorption through the whole SMB, instead of using few hundreds of parallel NCSTR models to represent each adsorber, the model described by Zwietering (1959) with side exits can be adapted. The modification relies on the use of a cascade of CSTR with side exits (MENCSTR), instead of the PFR Zwietering used to describe a completely segregated system. The two free flow zones are then added to the previous model by linking the side exits to a second NCSTR model in different inlet points (2MENCSTR). Hence, a fine 1D description of the SMB bed hydrodynamics is obtained. In our next paper, this description will be coupled with multicomponent adsorption and used to study the impact of the bed geometry on the quality of the separation, regardless of the quality of the adsorbent particles. The breakthrough curves obtained with the 2MENCSTR model will be compared with those obtained through CFD. This model converges in seconds contrasting with CFD simulations that can take up to three days and would rend its use on cyclic multi-bed simulations impracticable.

\section{APPENDIX A}

Deduction of the degree of mixing of a cascade of CSTR with the same residence time

The mean $\left(\mu_{1}\right)$ and the variance $\left(\sigma^{2}\right)$ of a cascade of CSTR can be obtained as a function of the residence time of one CSTR $\tau$, and the number of enchained CSTR $N$ (Warnecke et al., 1985):

$\mu_{1}=\tau N$

$\sigma^{2}=\tau^{2} N$ 
Thus, through the definition of variance:

$\sigma^{2}=m_{2}-m_{1}^{2}$

$\Leftrightarrow m_{2}=\tau^{2}(N+1) N$

With this it is possible to obtain the first two raw moments in every enchained CSTR, by replacing $N$. by the position of the CSTR in the chain, in Eqs. (A.1) and (A.3). The volume average of these two raw moments is equal to their sum over all the reactors and divided by $N$ :

$\bar{\mu}_{1, V}=\bar{m}_{1, V}=\frac{\sum_{i=1}^{N} i \tau}{N}=\frac{\tau}{2}(N+1)$

$\bar{m}_{2, V}=\frac{\sum_{i=1}^{N} i(i+1) \tau^{2}}{N}=\frac{\tau^{2}}{3}(N+1)(N+2)$

The volume average of the square of the first moment $\overline{m_{1, V}^{2}}$, can be obtained using:

$\overline{m_{1, V}{ }^{2}}=\frac{\sum_{i=1}^{N}(i \tau)^{2}}{N}=\frac{\tau^{2}}{6}(N+1)(2 N+1)$

Replacing Eqs. (A.4), (A.5) and (A.6) in Eq. (5):

$J=\frac{\frac{\tau^{2}}{6}(N+1)(2 N+1)-\left[\frac{\tau}{2}(N+1)\right]^{2}}{\frac{\tau^{2}}{3}(N+1)(N+2)-\left[\frac{\tau}{2}(N+1)\right]^{2}} \Leftrightarrow J=\frac{N-1}{N+5}$

For high $N$, the cascade of CSTR behaves similarly to a DPF with a Peclet number equal to $2 N$ (Schweich, 2001), and the degree of mixing of a DPF is given by:

$J=\frac{P e-2}{P e+10}$

\section{APPENDIX B}

In order to fit the 2MENCSTR model, the transport of the first two moments was performed through Eq. (6) in each zone of the system and not on the complete system composed by the three zones (the zone $A$ is the free flow zone above the porous bed, zone $P$ the porous media and the zone $B$ is the free flow zone below the porous media) as shown in Fig. B.1 a). 
The width of the considered geometry is discretized in $\omega_{T}$ parts, equal in terms of fluid flux. In a first step, any trajectory inside the three zones is modelled as a set of 3 cascades of N CSTR, one for each zone, as shown in Fig. B.1 b). The number of CSTR inside each zone is deduced from the previously calculated moments. Such approximation is possible since the degree of mixing in zones $A$ and $P$ is close to 1 , indicating that our system is close to complete segregation.

Since zone $\mathrm{A}$ is almost non-dispersive $\left(\left(m_{2}-m_{1}^{2}\right)_{A}=0.03 \mathrm{~s}^{2}\right)$ and the degree of mixing of zone $\mathrm{A}$ and $\mathrm{P}$ is high $(J \approx 1)$, zone $\mathrm{A}$ behaves like a delay region upstream the adsorption bed and can be modelled together with zone B, downstream of zone P, as shown in Fig. B.1 c). The assembly of zones A and B is modelled as a set of parallel NCSTR models downstream of zone P, and $\tau$ and $N$ are adjusted to the first two moments of these zones.
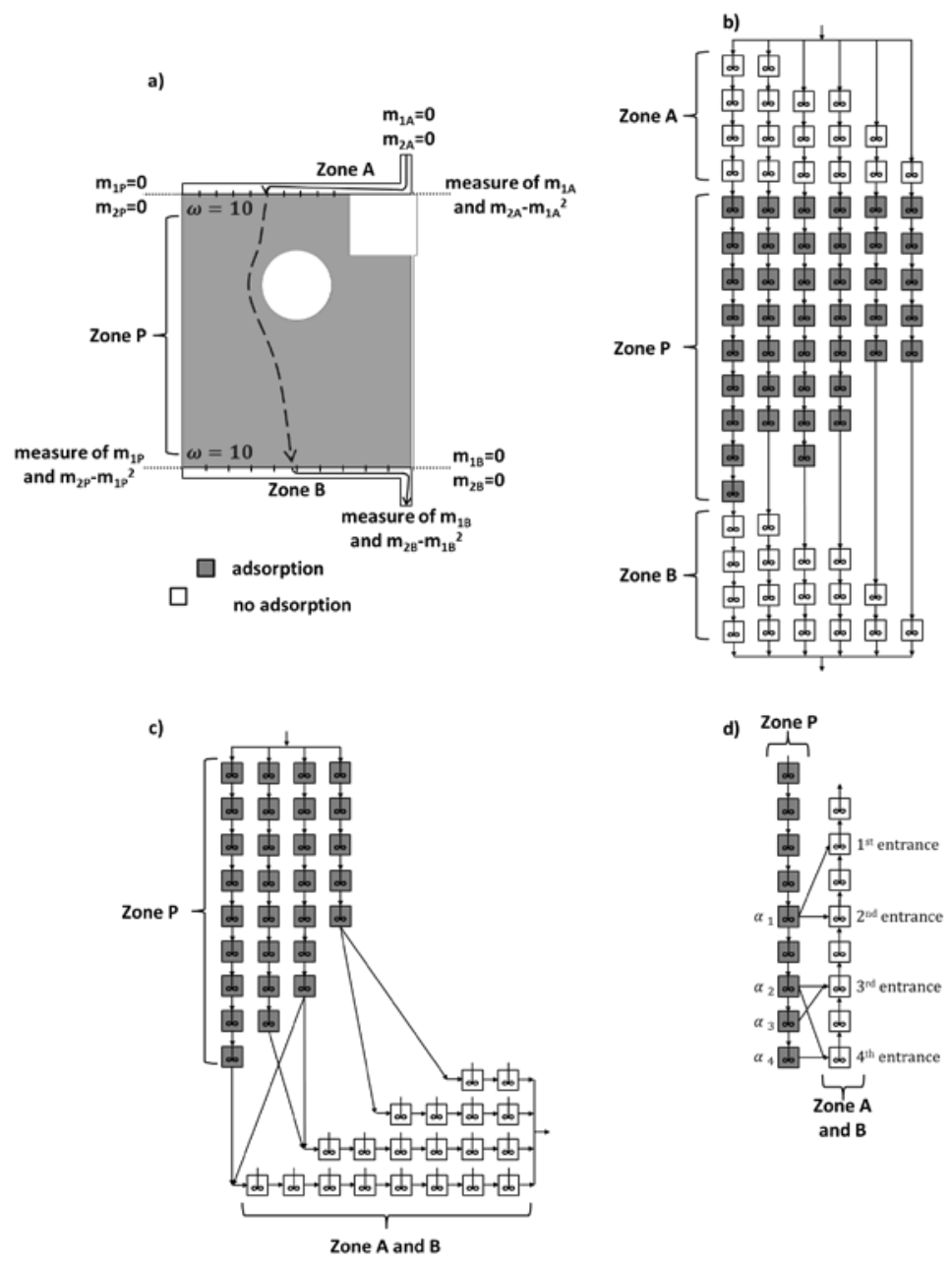

Fig. B.1 - Scheme of the modelling approach proposed (a), resulting system of parallel NCSTR models with the distinction of zone $P(b)$, simplification by modelling the zone A downstream the zone $P(c)$ and resulting 2MENCSTR model (d).

Another aspect visible in the transition from Fig. B.1 b) to $c$ ) is that the NCSTR models that are analogous (in zone $P$ and zone $A+B$ ) can be merged together in order to simplify the system. In the example of Fig. B.1 this simplification can decrease the number of parallel NCSTR models from 6 to 4 . Because of this, there are two NCSTR in zone $P$ that are split in order to be linked to different NCSTR of the zone $A+B$.

Finally, by looking at Fig. B.1 c), the longest NCSTR model in each zone (zone P and zone A+B) can be rebuilt as an extension of the shorter ones. A given NCSTR model can be rebuilt by picking a 
shorter NCSTR model and adding $N$ CSTR of equal residence $\tau$. N and $\tau$ are adjusted in order to fit residence time and dispersion of the NCSTR model of interest. By doing this separately for zone $P$ and zone $A+B$, we represent the first zone with a Multiple Exit NCSTR model and the second zone with a Multiple Entrance NCSTR model, resulting in what we denominate the 2MENCSTR model (as shown in Fig. B.1 d).

The following factors were imposed in order to simplify the development of the 2MENCSTR:

- The number of points with side exits of the NCSTR representing zone $P$ is imposed and equal to the number of points with side entrances of the NCSTR that represents zone A+B.

- The variation of the first moment between the side exit points is imposed to be constant. The same applies for the side entrance points.

- In the NCSTR model that represents zone A+B the number of CSTR between side entrances is also set constant and adjusted to the sum of the dispersion of zones A and B.

\section{NOTATIONS}

C concentration $\left(\mathrm{mol} \mathrm{m}^{-3}\right)$

$C_{\varepsilon 1} \quad k-\varepsilon$ parameter (dimensionless)

$C_{\varepsilon 2} \quad k-\varepsilon$ parameter (dimensionless)

$C_{\mu} \quad k$ - $\varepsilon$ parameter (dimensionless)

$D \quad$ overall diffusivity coefficient $\left(\mathrm{m}^{2} \mathrm{~s}^{-1}\right)$

$D_{M} \quad$ molecular diffusion coefficient $\left(\mathrm{m}^{2} \mathrm{~s}^{-1}\right)$

$D_{P} \quad$ mechanical dispersion coefficient $\left(\mathrm{m}^{2} \mathrm{~s}^{-1}\right)$

$d_{p} \quad$ particle diameter $(\mathrm{m})$

$D_{T} \quad$ turbulent diffusivity coefficient $\left(\mathrm{m}^{2} \mathrm{~s}^{-1}\right)$

$E(t)$ exit age $\left(\mathrm{s}^{-1}\right)$

$J \quad$ degree of mixing (dimensionless)

$K \quad$ bed permeability $\left(\mathrm{m}^{2}\right)$

$k \quad$ turbulent kinetic energy $\left(\mathrm{m}^{2} \mathrm{~s}^{-2}\right)$

$L_{e} \quad$ vessel outlet length in 2D space $(\mathrm{m})$

$m_{n} \quad$ raw moment of order $n\left(s^{n}\right)$

$N$ number of equivalent CSTR (dimensionless)

$n \quad$ order of the moment (dimensionless)

$p$ pressure $(\mathrm{Pa})$

$P e \quad$ Peclet number $\left(=v d_{p} / D_{P}\right)$

$P_{k} \quad k-\varepsilon$ term $\left(\mathrm{kg} \mathrm{m}^{-1} \mathrm{~s}^{-3}\right)$

$R e_{p} \quad$ particle Reynolds number $\left(=d_{p} v \rho / \mu\right)$

$S c_{T} \quad$ turbulent Schmidt number $\left(=\mu / \rho D_{T}\right)$

$u \quad$ superficial liquid velocity $\left(\mathrm{m} \mathrm{s}^{-1}\right)$

\section{Greek letters}

$\alpha \quad$ number of 2MENCSTR linking points

$\beta \quad$ non-Darcy coefficient $\left(\mathrm{m}^{-1}\right)$

$\varepsilon \quad$ bed porosity (dimensionless)

$\varepsilon_{T} \quad$ turbulent dissipation rate $\left(\mathrm{m}^{2} \mathrm{~s}^{-3}\right)$

$\mu \quad$ dynamic viscosity ( $\mathrm{Pa} \mathrm{s}$ )

$\mu_{e} \quad$ effective dynamic viscosity (Pa s)

$\mu_{n} \quad$ centred moment of order $n\left(\mathrm{~s}^{\mathrm{n}}\right)$

$\mu_{T} \quad$ turbulent dynamic viscosity (Pa s)

$\omega_{T} \quad$ number of mesh elements

$\rho$ mass density $\left(\mathrm{kg} \mathrm{m}^{-3}\right)$

$\sigma^{2}$ variance $\left(\mathrm{s}^{2}\right)$

$\sigma_{\varepsilon} \quad k-\varepsilon$ parameter (dimensionless) 
$\sigma_{k} \quad k-\varepsilon$ parameter (dimensionless)

$\tau \quad$ CSTR residence time (s)

Indices

$\begin{array}{ll}A & \text { above } \\ a x & \text { axial } \\ B & \text { below } \\ e & \text { exit } \\ n & \text { of order } \mathrm{n} \\ t r & \text { transversal } \\ V & \text { volume average }\end{array}$

Abbreviations

2MENCSTR Multiple Entrances and Multiple Exits NCSTR models

CFD Computational Fluid Dynamics

CSTR Continuous Stirred-tank Reactor

DPF Dispersed Plug Flow

MENCSTR Multiple Exits NCSTR model

NCSTR Cascade of N CSTR

PDF Probability Density Function

PFR Plug Flow Reactor

PNCSTR Set of Parallel NCSTR models

RANS Reynolds-averaged Navier-Stokes

RTD Residence Time Distribution

SMB Simulated Moving Bed

\section{REFERENCES}

Augier, F., Hotier, G., 2011. U.S. Patent Application 13/154,874.

Augier, F., Laroche, C., Brehon, E., 2008. Application of computational fluid dynamics to fixed bed adsorption calculations: effect of hydrodynamics at laboratory and industrial scale. Separation and Purification Technology, 63(2), 466-474.

Baléo, J. N., Le Cloirec, P., 2000. Validating a prediction method of mean residence time spatial distributions. AIChE journal, 46(4), 675-683.

Benyahia, F., 2004. On the modeling of flow in packed bed systems. Particulate science and technology, 22(4), 367-378.

Bergeot, G., Leinekugel-Le-Cocq, D., Wolff, L., Muhr, L., Bailly, M., 2010. Intensification of paraxylene production using a simulated moving bed reactor. Oil \& Gas Science and TechnologyRevue d'IFP Energies nouvelles, 65(5), 721-733.

Chan, E. C., Lien, F. S., Yovanovich, M. M., 2000. Numerical study of forced flow in a back-step channel through porous layer. In 2000 National Heat Transfer Conference, Pittsburgh, PA.

Claudel, S., Fonteix, C., Leclerc, J. P., Lintz, H. G., 2003. Application of the possibility theory to the compartment modelling of flow pattern in industrial processes. Chemical engineering science, 58(17), 4005-4016.

Combest, D. P., Ramachandran, P. A., Dudukovic, M. P., 2011. On the gradient diffusion hypothesis and passive scalar transport in turbulent flows. Industrial \& Engineering Chemistry Research, 50(15), 8817-8823. 
Danckwerts, P. V., 1953. Continuous flow systems: distribution of residence times. Chemical Engineering Science, 2(1), 1-13.

Danckwerts, P. V., 1958. The effect of incomplete mixing on homogeneous reactions. Chemical Engineering Science, 8(1), 93-102.

Delgado, J. M. P. Q., 2006. A critical review of dispersion in packed beds. Heat and mass transfer, 42(4), 279-310.

Ergun, S., 1952. Fluid flow through packed columns. Chem. Eng. Prog., 48, 89-94.

Flesch, T. K., Prueger, J. H., Hatfield, J. L., 2002. Turbulent Schmidt number from a tracer experiment. Agricultural and Forest Meteorology, 111(4), 299-307.

Foumeny, E. A., Chowdhury, M. A., McGreavy, C., Castro, J. A., 1992. Estimation of dispersion coefficients in packed beds. Chemical engineering \& technology, 15(3), 168-181.

Guo, B., Yu, A., Wright, B., Zulli, P., 2006. Simulation of turbulent flow in a packed bed. Chemical engineering \& technology, 29(5), 596-603.

Hocine, S., Pibouleau, L., Azzaro-Pantel, C., Domenech, S., 2008. Modelling systems defined by RTD curves. Computers \& Chemical Engineering, 32(12), 3112-3120.

John, V., Angelov, I., Öncül, A. A., Thévenin, D., 2007. Techniques for the reconstruction of a distribution from a finite number of its moments. Chemical Engineering Science, 62(11), 2890-2904.

Jones, W. P., Launder, B., 1972. The prediction of laminarization with a two-equation model of turbulence. International journal of heat and mass transfer, 15(2), 301-314.

Jongen, T., 2004. Extension of the age of fluid method to unsteady and closed AIChE journal, 50(9), 2020-2037.

Koeltzsch, K., 2000. The height dependence of the turbulent Schmidt number within the boundary layer. Atmospheric Environment, 34(7), 1147-1151.

Kwapinski, W., Salem, K., Mewes, D., \& Tsotsas, E., 2010. Thermal and flow effects during adsorption in conventional, diluted and annular packed beds. Chemical Engineering Science, 65(14), 4250-4260.

Laroche, C., Leinekugel-le-Cocq, D., Leflaive, P., 2011. Variable desorbent strength: influence on SMB operating conditions and performance. Adsorption, 17(1), 39-48.

Launder, B. E., Spalding, D. B., 1974. The numerical computation of turbulent flows. Computer methods in applied mechanics and engineering, 3(2), 269-289.

Liu, M., 2012. Age distribution and the degree of mixing in continuous flow stirred tank reactors. Chemical Engineering Science, 69(1), 382-393.

Liu, M., Tilton, J. N., 2010. Spatial distributions of mean age and higher moments in steady continuous flows. AIChE journal, 56(10), 2561-2572.

Minceva, M., Rodrigues, A. E., 2007. Understanding and revamping of industrial scale SMB units for p-xylene separation. AIChE journal, 53(1), 138-149.

Moustiri, S., Hebrard, G., Thakre, S. S., Roustan, M., 2001. A unified correlation for predicting liquid axial dispersion coefficient in bubble columns. Chemical engineering science, 56(3), 1041-1047.

Nield, D. A., 1991. The limitations of the Brinkman-Forchheimer equation in modeling flow in a saturated porous medium and at an interface. International Journal of Heat and Fluid Flow, 12(3), 269272.

Ruthven, D. M., Ching, C. B., 1989. Counter-current and simulated counter-current adsorption separation processes. Chemical Engineering Science, 44(5), 1011-1038. 
Sandberg, M., 1981. What is ventilation efficiency?. Building and environment, 16(2), 123-135.

Schweich, D. (Ed.)., 2001. Génie de la réaction chimique. Tec \& Doc.

Simcik, M., Ruzicka, M. C., Mota, A., Teixeira, J. A., 2012. Smart RTD for multiphase flow systems. Chemical Engineering Research and Design, 90(11), 1739-1749.

Spalding, D. B., 1958. A note on mean residence-times in steady flows of arbitrary complexity. Chemical Engineering Science, 9(1), 74-77.

Vafai, K., Kim, S. J., 1995. On the limitations of the Brinkman-Forchheimer-extended Darcy equation. International Journal of Heat and Fluid Flow, 16(1), 11-15.

Vafai, K., Tien, C. L., 1982. Boundary and inertia effects on convective mass transfer in porous media. International Journal of Heat and Mass Transfer, 25(8), 1183-1190.

Van Baten, J. M., Ellenberger, J., Krishna, R., 2001. Radial and axial dispersion of the liquid phase within a KATAPAK-S ${ }^{\circ}$ structure: experiments vS. CFD simulations. Chemical Engineering Science, 56(3), 813-821.

Warnecke, H. J., Prüss, J., Langemann, H., 1985. On a mathematical model for loop reactors-I. Residence time distribution, moments and eigenvalues. Chemical engineering science, 40(12), 23212326.

Yablonsky, G. S., Constales, D., Marin, G. B., 2009. A new approach to diagnostics of ideal and non-ideal flow patterns: I. The concept of reactive-mixing index (REMI) analysis. Chemical Engineering Science, 64(23), 4875-4883.

Zeng, Z., Grigg, R., 2006. A criterion for non-Darcy flow in porous media. Transport in Porous Media, 63(1), 57-69.

Zheng, X., Liu, Y., \& Liu, W., 2010. Two-dimensional modeling of the transport phenomena in the adsorber during pressure swing adsorption process. Industrial \& Engineering Chemistry Research, 49(22), 11814-11824.

Zwietering, T. N., 1959. The degree of mixing in continuous flow systems. Chemical Engineering Science, 11(1), 1-15. 